๑) Open Access Full Text Article

ORIGINAL RESEARCH

\title{
Assessment of Mesiobuccal Canal Configuration, Prevalence and Inter-Orifice Distance at Different Root Thirds of Maxillary First Molars: A CBCT Study
}

This article was published in the following Dove Press journal:

Clinical, Cosmetic and Investigational Dentistry

Mey Al-Habib

Mohammed Howait (D)

Department of Endodontics, Faculty of Dentistry King Abdul Aziz University, Jeddah, Saudi Arabia
Correspondence: Mohammed Howait P.O. Box 80209, Jeddah, 21589, Saudi Arabia

Email Mhowait@kau.edu.sa

\begin{abstract}
Aim: The aims of this study were to assess the prevalence and configuration of the second mesiobuccal (MB2) canal in the mesiobuccal MB root of permanent maxillary first molars at different root levels in a Saudi sub-population using cone-beam computed tomography $(\mathrm{CBCT})$ and to measure the inter-orifice distance between MB \& MB2 at the pulpal floor level.

Materials and Methods: This retrospective study was carried out on CBCT images of Saudi patients seen at King Abdul Aziz University. A total of 106 maxillary first molars were evaluated using a specialized software program (iCAT CBCT software). The prevalence of MB2, canal configuration and location of joining (apical-middle-coronal), if present was calculated. Also, the inter-orifice distance between both canals was measured at the level of the pulpal floor. Chi-square and one-way analysis of variance (ANOVA) were used.

Results: The MB2 canal was found in $92(86.8 \%)$ maxillary first molar teeth. The MB and MB2 canals were joined in 61 (58\%) cases, where the location of joining was $14(23 \%), 17$ $(27 \%)$, and $31(50 \%)$ in the coronal, middle, and apical third, respectively. The mean interorifice distance between both canals at the level of the pulpal floor was $2.52 \pm 0.76 \mathrm{~mm}$.

Conclusion: The prevalence of MB2 canal in this Saudi sub-population was high. The prevalence decreased as the root canal approached the apical third. There was no correlation between the average distance and occurrence of canal joining. CBCT is useful in detecting and mapping the mesiobuccal root canal system, with a likelihood of enhancing the quality of root canal treatment.
\end{abstract}

Keywords: CBCT, maxillary first molar, root canal anatomy, mesiobuccal root morphology

\section{Introduction}

To achieve the main objectives of endodontic treatment, all root canals need to be identified, accessed, cleaned, shaped, and filled. ${ }^{1,2}$ Hence, it is crucial to understand the anatomy of the root canal system and the prevalence of frequent variations. ${ }^{1}$ It has been reported that maxillary first molars usually have three roots with three to four canals. ${ }^{3}$ Several methods of examining the anatomy of the mesiobuccal (MB) root of the maxillary first molar have been reported in the literature. Some of these methods include clinical, radiographical, and histological investigations such as clearing, staining, and sectioning. ${ }^{4,5}$ More recently, investigators have begun using cone-beam computed tomography $(\mathrm{CBCT})$ to measure the prevalence of the second 
mesiobuccal canal (MB2) and the type of canal configuration. ${ }^{6,7}$ Anatomical studies have reported the prevalence of the MB2 canal in the mesial root of the maxillary first and second molar to be between 69 to $93 \%$. $^{6,8-12}$

The complex root and canal anatomy of maxillary molars lie behind the high rates of endodontic treatment failure observed for these teeth. It has been hypothesized that failure to find the MB2 canal is the main cause of reduced long-term prognosis after root canal treatment in maxillary molars. ${ }^{13,14}$ The prevalence of endodontic treatment failure due to untreated MB2 canal as reported in the literature ranges from $46 \%$ to $78 \% .{ }^{15-17}$ This shows the importance of locating and treating the MB2 canal.

The most commonly used technique to identify the anatomy of root canals is a periapical radiograph, which provides limited details of the root canal anatomy., ${ }^{4,18,19}$ CBCT, however, can generate 3-D images with higher resolution and lower radiation dose than conventional computed tomography (CT). ${ }^{20,21}$

It has been reported that $\mathrm{MB} 2$ detection using $\mathrm{CBCT}$ is more reliable than clinical visual inspection. ${ }^{21} \mathrm{CBCT}$ images have become an important aid in identifying MB2 by providing anatomical information, avoiding unnecessary tooth preparation, and reducing the risk of untoward incidents such as instrument separation, canal transportation, and perforation. ${ }^{4,22,23}$ Specific guidelines have been issued by the American Association of Endodontists and American Academy of Oral and Maxillofacial Radiology justifying the use of CBCT imaging in endodontics specifically for teeth with complex root canal anatomy systems. ${ }^{24}$

Certain aspects of the root and canal morphology of permanent maxillary first molars in the western Saudi Arabian subpopulation revealed by CBCT have not been reported previously in the literature. Thus, the aim of this study was to use CBCT to assess the prevalence and configuration of the MB2 canal in the MB root of permanent maxillary first molars in a Saudi population at different root levels and to measure the inter-orifice distance between MB and MB2 at the level of the pulpal floor.

\section{Materials and Methods}

This retrospective study was conducted at the Faculty of Dentistry at King Abdul Aziz University. The study protocol was approved by the Research Ethics Committee of the Faculty of Dentistry at King Abdul Aziz University (No. 02-02-20) and all participants signed an informed consent that their data will anonymously be used for research purposes. The present study complied with the principles of the Declaration of Helsinki. A total of 106 maxillary first molars were included in this study (44 males and 62 females) with an age range of 20 to 65 years. The sample was divided into four age groups (20$30,31-40,41-50,51-65)$. The CBCT scans had been taken earlier for various diagnostic purposes such as implant planning, surgical removal of impacted teeth and endodontic evaluations. Teeth were selected if they met the following inclusion criteria: no periapical lesions, resorption, or canal calcification, no previously root canal treated or previously initiated root canal teeth, and no cemented posts or large coronal restorations.

All patients were imaged with an iCAT scanner (Imaging Sciences International, Hatfield, PA, USA); $8 \times$ $8 \mathrm{~cm}$ images were scanned at $120 \mathrm{KVp}$ and 5-7 mA using voxel size $0.2 \mathrm{~mm}$. Images were displayed on a calibrated Dell (Round Rock, TX) 17 inch (0.28 dot pitch) highresolution color monitor set at a 16-bit color depth and a screen resolution of $1024 \times 768$ pixels. Two calibrated examiners, two certified endodontists (examiner 1,2), reviewed the CBCT scans using Vision software (Imaging Science International, Hatfield, PA, USA). Before evaluation of the CBCT images, an adequate review of image processing and reconstruction was performed and criteria for reading and recording data were set. After intra-examiner calibration, the two examiners separately evaluated the images in the study samples. If a disagreement on the interpretation of an image occurred, it was first discussed between the two examiners. When a consensus could not be reached, a radiologist assisted in reaching a decision. The images were reviewed in a dimly lit room with no windows. After adjusting the image contrast and using the magnification tool for optimal visualization, all 106 permanent maxillary first molars were evaluated. The presence or absence of MB2 and the location where $\mathrm{MB}$ and MB2 join (coronal, middle or apical) were also recorded. The distance between both canals was measured at the level of the pulpal floor. The examination was performed using a multi-planar view, mainly the axial view, by scrolling through the sections from the canal orifice to the root apex several times searching for the MB2 canal. When the presence of MB2 was identified, the distance between MB and MB2 was measured. Measurement of the whole root was done and divided equally into thirds (coronal, middle, or apical). The joining of MB and MB2 canals was determined by 
Table I The Prevalence of MB2 Canals According to Gender

\begin{tabular}{|c|c|c|c|c|}
\hline Gender & Sample Number n (\%) & MB2 Present n (\%) & MB2 Absent n (\%) & P-value \\
\hline Male & $44(4 \mid .5)$ & $4 \mathrm{I}(93.2)$ & $3(6.8)$ & \multirow[t]{3}{*}{0.102} \\
\hline Female & $62(58.5)$ & $51(82.3)$ & II (I7.7) & \\
\hline Total & $106(100)$ & $92(86.8)$ & $14(13.2)$ & \\
\hline
\end{tabular}

Table 2 The Prevalence of MB2 Canals According to Age

\begin{tabular}{|l|c|c|c|c|}
\hline Age Group & Sample Number & MB2 Present (\%) & MB2 Absent (\%) & P-value \\
\hline $20-30$ & 46 & 41 & 5 & 0.162 \\
$3 \mathrm{I}-40$ & 36 & 32 & 4 & 3 \\
$4 \mathrm{I}-50$ & 20 & 17 & 2 & \\
$5 \mathrm{I}-65$ & 4 & 2 & $14(13.2)$ & \\
Total $\mathrm{n}(\%)$ & $106(100)$ & $92(86.8)$ & \\
\hline
\end{tabular}

identifying the location of joining at root one-third levels, coronal, middle or apical.

Descriptive statistics were generated for the configuration and presence of the MB2 canal and the distance between $\mathrm{MB}$ and $\mathrm{MB} 2$ in the maxillary first molars. Intra- and inter-examiner reliability data were analyzed using Kappa tests. The SPSS for Windows software (ver. 22.0; SPSS Inc., Chicago, IL, USA) was used to conduct the statistical tests using the Pearson chi-square test and one-way analysis of variance (ANOVA). Statistical significance was set at $(\mathrm{P}<0.05)$.

\section{Results}

The intra-examiner reliability was 0.87 and 0.85 for examiner 1 and 2 respectively, and the inter-examiner reliability was 0.82 . The study involved 106 maxillary first molars including $44(41.5 \%)$ males and 62 (58.5\%) females with an average age of $40 \pm 11.76$ and an age range of 20 to 65 years (Tables 1 and 2). A second mesiobuccal canal was found in 92 teeth $(86.8 \%)$ maxillary first molar teeth (41 males, 51 females) as shown in Table 1. Representative CBCT images showing different mesiobuccal root canal configurations are shown in Figure 1.

A Pearson chi-square test showed no statistically significant correlation between the prevalence of MB2 and gender $(P=0.102)$ (Table 1$)$, or age group $(P=0.162)$ (Table 2). When comparing the bilateral symmetry of the number of roots and canals, all patients included in the sample had both right and left maxillary first molars present. We found that $92 \%$ of the sample had a similar number of roots and canal configurations on both sides.
The joining between MB and MB2 occurred in 61 teeth (58\% of cases) (Table 3 ) and the location of joining was 14 (23\%), 17 (27\%), and 31 (50\%) coronal, middle, and apical third, respectively (Table 3 ). ANOVA showed that there was no statistically significant difference between the locations of joining at different root thirds $(P=0.19)$. The mean inter-orifice distance between both canals at the level of the pulpal floor was $2.52 \pm 0.76 \mathrm{~mm}$ (Figure 2).

\section{Discussion}

The objectives of this study were to assess the prevalence and configuration of a second mesiobuccal canal in the $\mathrm{MB}$ root of permanent maxillary first molars in the western Saudi sub-population using CBCT, to determine the location where MB2 joins MB at different root levels and to measure the mean distance between both MB and MB2 canal orifice at the level of the pulpal floor which has not been reported in the literature previously.

Our results show that the prevalence of the MB2 canal in the maxillary first molars of Western Saudi sub-population is $86.8 \%$. This finding is in close agreement with several international anatomical studies that showed a prevalence between $69 \%$ and $90 \% .^{6,8-11}$ Other studies have reported the morphology and canal anatomy of the mesiobuccal root (MBR) in maxillary first molars in the Saudi Arabian population specifically. ${ }^{25-28}$ Al-Nazhan ${ }^{25}$ found that only $23.3 \%$ of their sample had two canals while Al-Fouzan et al reported a higher MB2 Prevalence of $51.3 \%{ }^{27}$ These studies showed a lower prevalence of MB2 than our finding. The difference is most likely due to different methods used in those study such as clinical and 
A

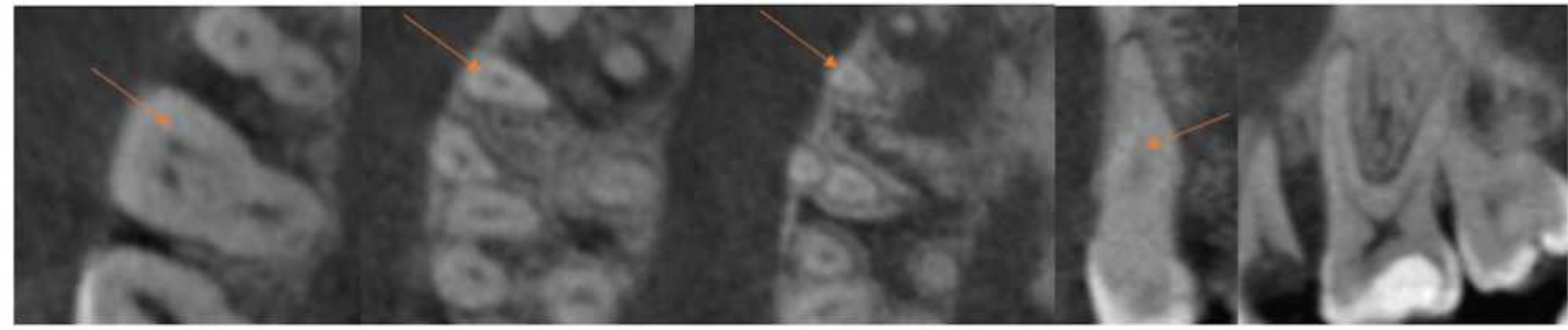

B

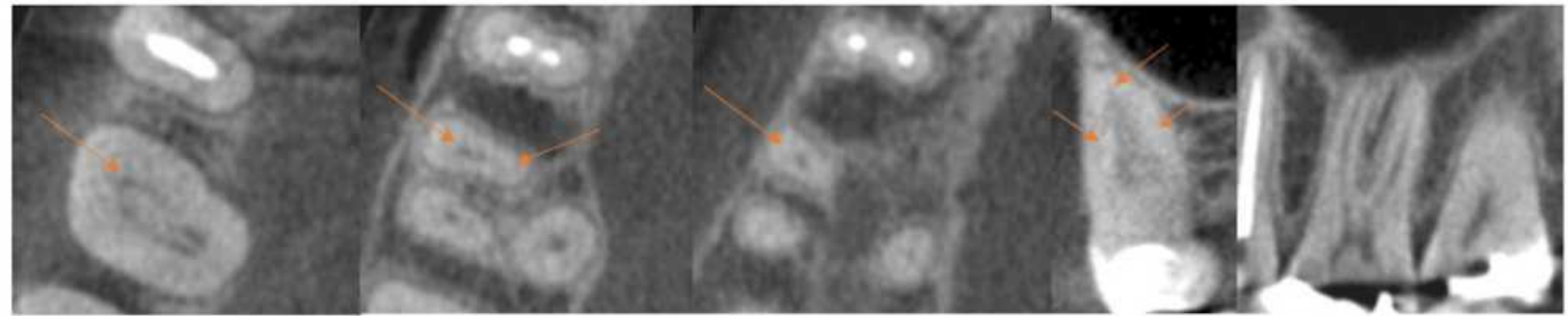

C

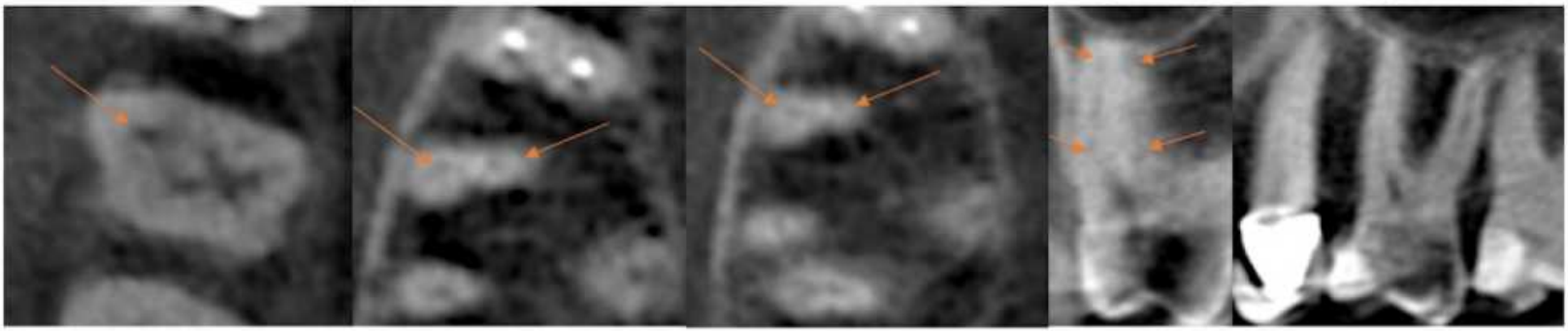

Axial

Coronal
Axial

Middle
Axial Apical
Sagittal

\section{Coronal}

Figure I Representative CBCT images showing mesiobuccal root canal configurations. Arrows represent position of the mesiobuccal canals in axial sections (coronal, middle, apical thirds), sagittal and coronal sections (left to right). (A) Vertucci type I. (B) Vertucci type II. (C) Vertucci type IV.

radiographic evaluation using optical magnification and/or periapical radiographs only. The use of conventional radiographs to study tooth morphology has the limitation by showing the object in two-dimensional views instead of in three dimensions. CBCT evaluation used in our study detects canal configuration at different root levels more accurately. On the other hand, when CBCT was used as the method of evaluation, a higher prevalence of the MB2 canal has been reported $\left(55.6 \%^{26}\right.$ and $\left.70.6 \%{ }^{28}\right)$, but still lower than our finding. This may be due to differences in sample size or different radiographic interpretation and CBCT setting parameters ie, voxel setting of $0.3 \mathrm{~mm}$ ). The accuracy of detecting additional canals depends on the voxel dimension and contrast resolution used. Bauman et $\mathrm{al}^{22}$ reported that $\leq 0.2 \mathrm{~mm}$ voxel sizes are optimal for the detection of $\mathrm{MB}$ canals with better $\mathrm{CBCT}$ resolution. In our present study, the voxel size of the CBCT machine was set at $0.2 \mathrm{~mm}$, which offered good diagnostic performance. The higher prevalence of MB2 reported in the majority of CBCT studies, including ours, suggests that

Table 3 The Prevalence of MB2 Canal According to the Location of Joining in the MB Root

\begin{tabular}{|c|c|c|c|c|c|}
\hline & \multicolumn{3}{|c|}{ Vertucci Type II (MB+MB2 Joining) n (\%) } & Vertucci Type IV (MB+MB2 Not Joining) n (\%) & Total n (\%) \\
\hline & \multicolumn{3}{|c|}{$62(58.5)$} & \multirow[t]{4}{*}{$44(4 I .5)$} & \multirow[t]{4}{*}{$106(100)$} \\
\hline Joining Location & Coronal Third & Middle Third & Apical Third & & \\
\hline n (\%) & $14(23 \%)$ & $17(27 \%)$ & $31(50 \%)$ & & \\
\hline$P$-value & \multicolumn{3}{|c|}{0.19} & & \\
\hline
\end{tabular}




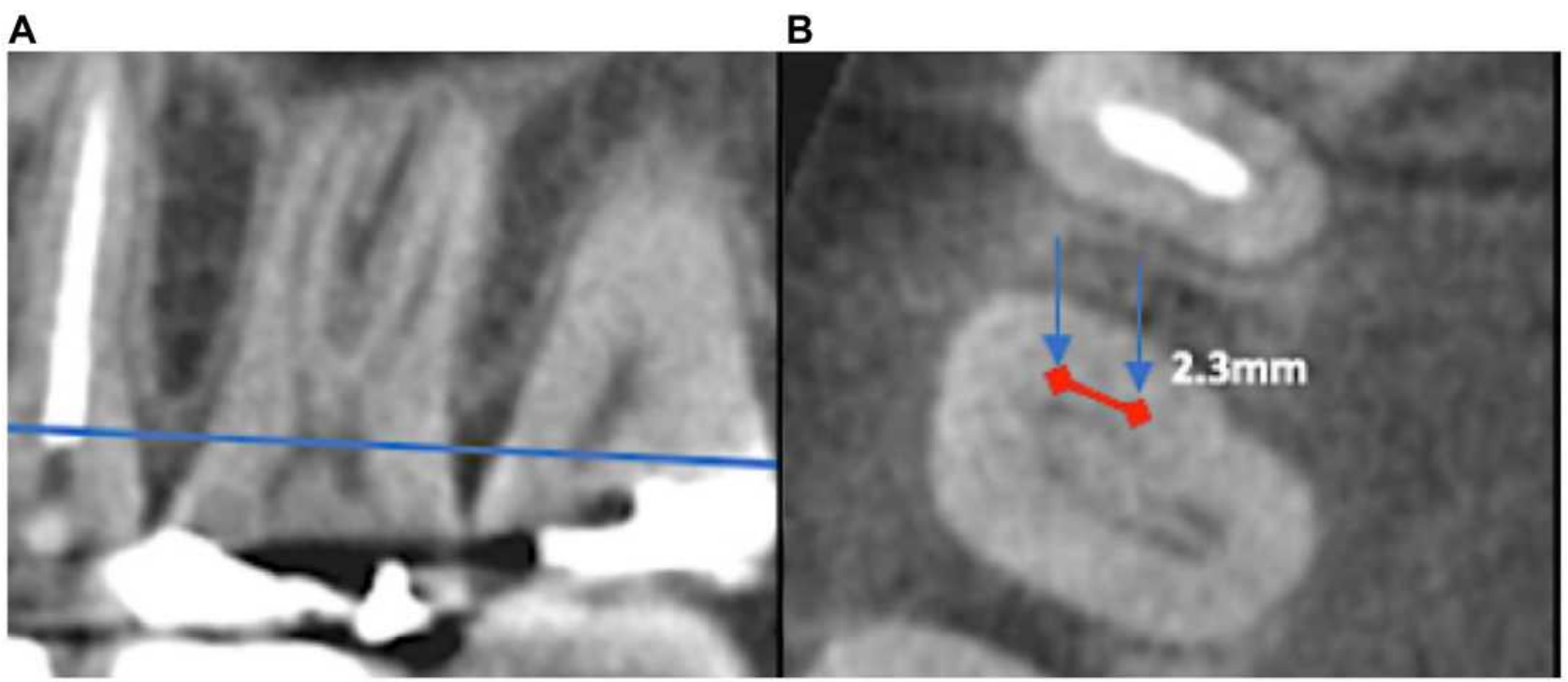

Figure 2 Representative CBCT images showing mesiobuccal root canals and inter-orifice distance at the pulpal floor level. (A) Sagittal section with a blue line representing the level of the pulpal floor. (B) Arrows representing the position of the mesiobuccal canals in an axial section at the level of the pulpal floor. Red line represents the interorifice distance measured at the pulpal floor level.

CBCT is an imaging modality aid that helps clinicians assess complex root canal morphology in a reliable manner. $^{29,30}$

CBCT scanning is a conservative tool that provides highly accurate $3 \mathrm{D}$ images with minimal distortion. It is considered an excellent method for identifying the internal anatomy of maxillary first molars and is a reliable method in the detection of the MB2 canal. $^{21,31}$ However, the amount of radiation dose and cost of $\mathrm{CBCT}$ relative to intraoral radiography, routine use of CBCT imaging may not be justifiable. It should be used as an adjunctive method when more information for example in cases of unusual canal anatomy or calcified canals.

CBCT has some limitations that may affect the quality of images and therefore may affect the detection of MB2. Those factors include high bone density, presence of restorations in the same arch, and movement of the patient during exposure. ${ }^{5}$ A significant issue that can impact the image interpretation and diagnostic accuracy of CBCT images is the scatter and beam hardening artifacts caused by high density adjacent structures, such as enamel or bone, and radiopaque materials such as metal posts, restorations and root filling materials. ${ }^{5}$

CBCT is also more effective in the mapping of the MB2 canal in all different levels of root structure: coronal, middle, apical. ${ }^{32}$ The possibility of joining of mesiobuccal canals increased moving from coronal to apical third with the same software program used in this study, which means that MB2 canals are less prevalent in the middle and apical thirds than in the coronal third. This finding is very helpful to the clinician when negotiating the MB2 canal during root canal treatment.

Our results demonstrate that the prevalence of MB2 canals decreases as the root canal approaches the apical third, which agrees with other studies in the Saudi population that reported a higher prevalence of Vertucci type I and II root canal configuration for $\mathrm{MB}$ canals of maxillary first molars in which there is a single canal or when MB2 joins the main canal, subsequently ending in one canal and one foramen. ${ }^{26}$ These findings have also been reported in Kuwaiti, Iraqi, Chinese, Japanese, and Brazilian populations. ${ }^{30,32-35}$

The mean distance between MB and MB2 canals at the level of the pulpal floor was $2.52 \pm 0.76 \mathrm{~mm}$. This finding gives insight to the clinician on where to locate the MB2 orifice relative to the $\mathrm{MB}$ orifice on the pulp floor in the Saudi sub-population. Su et $\mathrm{al}^{36}$ reported an average interorifice distance of $1.91 \pm 0.59 \mathrm{~mm}$ in the Taiwanese population while Moidu et $\mathrm{al}^{37}$ reported $2.58 \pm 0.04 \mathrm{~mm}$ in the Indian population.

In the present study, there was no correlation between gender and the presence of MB2. This result is in agreement with those of Reis et $\mathrm{al}^{32} \mathrm{Al}-\mathrm{Nazhan}{ }^{25}$ and Zhang et al. ${ }^{34}$ In contrast, MB2 was shown to be predominant in males, as also reported by Al-Shehri et $\mathrm{al}^{26}$ Faraj, $^{38}$ Ratanajirasut et $\mathrm{al}^{39}$ and Kim et $\mathrm{al}^{35}$ who found a relationship between gender and the 
number of canals. We also found no relationship between age and prevalence of MB2 canals. This is in agreement with the studies of Al-Shehri et $\mathrm{al}^{26}$ Ratanajirasut et $\mathrm{al}^{39}$ and Fogel et al. ${ }^{40}$ On the other hand, Reis et al found that the prevalence of MB2 canals decreases as age increases in a Brazilian population. $^{32}$ This decrease could be attributed to dentin deposition that can result in narrowing of the MB2 canal. $^{40}$

There are some limitations in our study, which include a small sample size and collection of the sample from one center. Also, our sample was limited to teeth with no previous root canal treatment, cemented posts or large coronal restorations, which was hard to collect since the first molar erupts at an early age and was usually found carious or restored by adulthood.

More accurate and reliable data may be generated by increasing the sample size and collecting it from different centers, leading to a stronger conclusion about the common morphological features and anatomical variations of maxillary first molars. Furthermore, the use of a limited view CBCT machine with lower voxel size will provide superior image quality, helping to explore the root and canal morphology more accurately. Moreover, cohort studies in which the same individuals are observed over time are necessary to analyze and describe factors such as age to determine whether the MB canals narrow or calcify into one canal and what age range impacts the number and size of the MB canals in maxillary molars.

\section{Conclusion}

A second mesiobuccal canal was present in $86.8 \%$ of maxillary first molars in the western Saudi sub-population. When analyzed with CBCT scanning, the prevalence of MB2 canals decreases as the root approaches the apical third. These results may help endodontists to understand the canal configuration of maxillary molars and improve the outcomes of their endodontic treatments.

\section{Ethics Approval and Informed Consent}

The study protocol was approved by the Research Ethics Committee of the Faculty of Dentistry at King Abdul Aziz University (No. 02-02-20) and all participants signed an informed consent that their data will anonymously be used for research purposes. The present study complied with the principles of the Declaration of Helsinki.

\section{Disclosure}

The authors report no conflicts of interest in this work.

\section{References}

1. Vertucci FJ. Root canal anatomy of the human permanent teeth. Oral Surg Oral Med Oral Pathol. 1984;58(5):589-599. doi:10.1016/00304220(84)90085-9

2. Weine FS, Healey HJ, Gerstein H, Evanson L. Canal configuration in the mesiobuccal root of the maxillary first molar and its endodontic significance. Oral Surg Oral Med Oral Pathol. 1969;28(3):419-425. doi:10.1016/0030-4220(69)90237-0

3. Pecora JD, Woelfel JB, Sousa Neto MD, Issa EP. Morphologic study of the maxillary molars. Part II: internal anatomy. Braz Dent J. 1992;3(1):53-57.

4. Baratto Filho F, Zaitter S, Haragushiku GA, de Campos EA, Abuabara A, Correr GM. Analysis of the internal anatomy of maxillary first molars by using different methods. J Endod. 2009;35 (3):337-342. doi:10.1016/j.joen.2008.11.022

5. Neelakantan P, Subbarao C, Subbarao CV. Comparative evaluation of modified canal staining and clearing technique, cone-beam computed tomography, peripheral quantitative computed tomography, spiral computed tomography, and plain and contrast medium-enhanced digital radiography in studying root canal morphology. $J$ Endod. 2010;36(9):1547-1551. doi:10.1016/j.joen.2010.05.008

6. Zheng QH, Wang Y, Zhou XD, Wang Q, Zheng GN, Huang DM. A cone-beam computed tomography study of maxillary first permanent molar root and canal morphology in a Chinese population. $J$ Endod. 2010;36(9):1480-1484. doi:10.1016/j.joen.2010.06.018

7. Omer OE, Al Shalabi RM, Jennings M, Glennon J, Claffey NM. A comparison between clearing and radiographic techniques in the study of the root-canal anatomy of maxillary first and second molars. Int Endod J. 2004;37(5):291-296. doi:10.1111/j.0143-2885.2 004.00731.x

8. Al Shalabi RM, Omer OE, Glennon J, Jennings M, Claffey NM. Root canal anatomy of maxillary first and second permanent molars. Int Endod J. 2000;33(5):405-414. doi:10.1046/j.1365-2591.20 00.00221.x

9. de Carvalho MC, Zuolo ML. Orifice locating with a microscope. $J$ Endod. 2000;26(9):532-534. doi:10.1097/00004770-20000900000012

10. Gilles J, Reader A. An SEM investigation of the mesiolingual canal in human maxillary first and second molars. Oral Surg Oral Med Oral Pathol. 1990;70(5):638-643. doi:10.1016/0030-4220(90) 90415-O

11. Imura N, Hata GI, Toda T, Otani SM, Fagundes MI. Two canals in mesiobuccal roots of maxillary molars. Int Endod J. 1998;31(6):410414. doi:10.1111/j.1365-2591.1998.0169.x

12. Martins JNR, Alkhawas MAM, Altaki Z, et al. Worldwide analyses of maxillary first molar second mesiobuccal prevalence: a Multicenter Cone-Beam Computed Tomographic Study. J Endod. 2018;44(11):1641-1649.e1641. doi:10.1016/j.joen.2018.07.027

13. Seidberg BH, Altman M, Guttuso J, Suson M. Frequency of two mesiobuccal root canals in maxillary permanent first molars. $J \mathrm{Am}$ Dent Assoc. 1973;87(4):852-856. doi:10.14219/jada.archive.19 73.0489

14. Yoshioka T, Kikuchi I, Fukumoto Y, Kobayashi C, Suda H. Detection of the second mesiobuccal canal in mesiobuccal roots of maxillary molar teeth ex vivo. Int Endod J. 2005;38(2):124-128. doi:10.1111/ j.1365-2591.2004.00918.x 
15. Wolcott J, Ishley D, Kennedy W, Johnson S, Minnich S, Meyers J. A $5 \mathrm{yr}$ clinical investigation of second mesiobuccal canals in endodontically treated and retreated maxillary molars. $J$ Endod. 2005;31 (4):262-264. doi:10.1097/01.don.0000140581.38492.8b

16. Nascimento EHL, Gaêta-Araujo H, Andrade MFS, Freitas DQ. Prevalence of technical errors and periapical lesions in a sample of endodontically treated teeth: a CBCT analysis. Clin Oral Investig. 2018;22(7):2495-2503. doi:10.1007/s00784-018-2344-y

17. Do Carmo WD, Verner FS, Aguiar LM, et al. Missed canals in endodontically treated maxillary molars of a Brazilian subpopulation: prevalence and association with periapical lesion using cone-beam computed tomography. Clin Oral Investig. 2020. doi:10.1007/ s00784-020-03554-4

18. Abuabara A, Baratto-Filho F, Aguiar Anele J, Leonardi DP, SousaNeto MD. Efficacy of clinical and radiological methods to identify second mesiobuccal canals in maxillary first molars. Acta Odontol Scand. 2013;71(1):205-209. doi:10.3109/00016357.2011.654262

19. Wolcott J, Ishley D, Kennedy W, Johnson S, Minnich S. Clinical investigation of second mesiobuccal canals in endodontically treated and retreated maxillary molars. $J$ Endod. 2002;28(6):477-479. doi:10.1097/00004770-200206000-00016

20. Patel S. New dimensions in endodontic imaging: part 2. Cone beam computed tomography. Int Endod J. 2009;42(6):463-475. doi:10.1111/j.1365-2591.2008.01531.x

21. Blattner TC, George N, Lee CC, Kumar V, Yelton CD. Efficacy of cone-beam computed tomography as a modality to accurately identify the presence of second mesiobuccal canals in maxillary first and second molars: a pilot study. $J$ Endod. 2010;36(5):867-870. doi:10.1016/j.joen.2009.12.023

22. Bauman R, Scarfe W, Clark S, Morelli J, Scheetz J, Farman A. Ex vivo detection of mesiobuccal canals in maxillary molars using CBCT at four different isotropic voxel dimensions. Int Endod $J$. 2011;44(8):752-758. doi:10.1111/j.1365-2591.2011.01882.x

23. Ibarrola JL, Knowles KI, Ludlow MO, McKinley IB Jr. Factors affecting the negotiability of second mesiobuccal canals in maxillary molars. J Endod. 1997;23(4):236-238. doi:10.1016/S0099-2399(97) 80054-4

24. Fayad MI, Nair M, Levin MD. AAE and AAOMR joint position statement: use of cone beam computed tomography in endodontics 2015 update. Oral Surg Oral Med Oral Pathol Oral Radiol. 2015;120 (4):508-512. doi:10.1016/j.0ooo.2015.07.033

25. Al-Nazhan S. The prevalence of two canals in mesial root of endodontically treated maxillary first molars among a Saudi Arabian subpopulation. Saudi Dent J. 2005;17:24-28.

26. Al-Shehri S, Al-Nazhan S, Shoukry S, et al. Root and canal configuration of the maxillary first molar in a Saudi subpopulation: a conebeam computed tomography study. Saudi Endod J. 2017;7(2):69-76.

27. Al-Fouzan KS, Ounis HF, Merdad K, Al-Hezaimi K. Incidence of canal systems in the mesio-buccal roots of maxillary first and second molars in Saudi Arabian population. Aust Endod J. 2013;39(3):98101. doi:10.1111/j.1747-4477.2010.00289.x

28. Alrahabi M, Sohail Zafar M. Evaluation of root canal morphology of maxillary molars using cone beam computed tomography. Pak J Med Sci. 2015;31(2):426-430. doi:10.12669/pjms.312.6753

Clinical, Cosmetic and Investigational Dentistry

\section{Publish your work in this journal}

Clinical, Cosmetic and Investigational Dentistry is an international, peer-reviewed, open access, online journal focusing on the latest clinical and experimental research in dentistry with specific emphasis on cosmetic interventions. Innovative developments in dental materials, techniques and devices that improve outcomes and patient
29. Ahmad I. Root and root canal morphology of Saudi Arabian permanent dentition. Saudi Endod J. 2015;5(2):99-106. doi:10.4103/16585984.155446

30. Al-Saedi A, Al-Bakhakh B, Al-Taee RG. Using cone-beam computed tomography to determine the prevalence of the second mesiobuccal canal in maxillary first molar teeth in a sample of an Iraqi population. Clin Cosmet Investig Dent. 2020;12:505-514. doi:10.2147/CCIDE. S281159

31. Matherne RP, Angelopoulos C, Kulild JC, Tira D. Use of cone-beam computed tomography to identify root canal systems in vitro. $J$ Endod. 2008;34(1):87-89. doi:10.1016/j.joen.2007.10.016

32. Reis AG, Grazziotin-Soares R, Barletta FB, Fontanella VR, Mahl CR. Second canal in mesiobuccal root of maxillary molars is correlated with root third and patient age: a cone-beam computed tomographic study. $J$ Endod. 2013;39(5):588-592. doi:10.1016/j. joen.2013.01.003

33. Pattanshetti N, Gaidhane M, Al Kandari AM. Root and canal morphology of the mesiobuccal and distal roots of permanent first molars in a Kuwait population-a clinical study. Int Endod J. 2008;41 (9):755-762. doi:10.1111/j.1365-2591.2008.01427.x

34. Zhang R, Yang H, Yu X, Wang H, Hu T, Dummer PM. Use of CBCT to identify the morphology of maxillary permanent molar teeth in a Chinese subpopulation. Int Endod J. 2011;44(2):162-169. doi:10.1111/j.1365-2591.2010.01826.x

35. Kim Y, Lee SJ, Woo J. Morphology of maxillary first and second molars analyzed by cone-beam computed tomography in a korean population: variations in the number of roots and canals and the incidence of fusion. $J$ Endod. 2012;38(8):1063-1068. doi:10.1016/j. joen.2012.04.025

36. Su CC, Wu Y-C, Chung MP, et al. Geometric features of second mesiobuccal canal in permanent maxillary first molars: a cone-beam computed tomography study. J Dent Sci. 2017;12(3):241-248. doi:10.1016/j.jds.2017.03.002

37. Moidu NP, Sharma S, Kumar V, Chawla A, Logani A. Association between the mesiobuccal canal configuration, interorifice distance, and the corresponding root length of permanent maxillary first molar tooth: a Cone-beam Computed Tomographic Study. J Endod. 2021;47 (1):39-43. doi:10.1016/j.joen.2020.08.025

38. Faraj BM. The frequency of the second mesiobuccal canal in maxillary first molars among a sample of the Kurdistan Region-Iraq population - A retrospective cone-beam computed tomography evaluation. J Dent Sci. 2021;16(1):91-95. doi:10.1016/j.jds.2020.06.021

39. Ratanajirasut R, Panichuttra A, Panmekiate S. A cone-beam computed tomographic study of root and canal morphology of maxillary first and second permanent molars in a Thai population. $J$ Endod. 2018;44(1):56-61. doi:10.1016/j.joen.2017.08.020

40. Fogel HM, Peikoff MD, Christie WH. Canal configuration in the mesiobuccal root of the maxillary first molar: a clinical study. $J$ Endod. 1994;20(3):135-137. doi:10.1016/S0099-2399(06)80059-2 satisfaction and preference will be highlighted. The manuscript management system is completely online and includes a very quick and fair peer-review system, which is all easy to use. Visit http://www.dovepress.com/testimonials.php to read real quotes from http://www.dovep
published authors.

\section{Dovepress}

